\title{
Frecuencia de hábitos bucales parafuncionales y su relación con el grupo etario.
}

\author{
Parafunctional oral habits frequency and their relationship with the age \\ group.
}

José Francisco Murrieta Pruneda ${ }^{1 a}$, Mariana Ramírez Márquez ${ }^{1}$, Luis Salgado Valdés ${ }^{1}$, Raquel Salamanca Torres ${ }^{1}$

\section{RESUMEN}

Objetivo: Comparar la frecuencia de hábitos orales disfuncionales y su relación con el grupo etario, ya sea infantil o preescolar.

Material y Métodos: Se llevó a cabo un estudio transversal en una muestra de 269 infantes y preescolares. La recolección de datos se basó en el examen bucal y la aplicación de un cuestionario (alfa de Cronbach $=0,832$ ), previa calibración de una Cirujana Dentista (Kappa $\geq 0,941$ ). Para medir la fiabilidad del cuestionario se calculó el valor del alfa de Cronbach y para las asociaciones la Chi cuadrada de Pearson.

Resultados. El hábito de morder objetos fue el hábito más frecuente $(31.2 \%)$, la onicofagia y la queilofagia mostraron diferencias estadísticamente significativas en relación con el grupo de preescolares $(p=0.004 ; p=0.023)$, mientras que el uso de biberón lo fue para el grupo de infantes $(p=0.0001)$.

Conclusiones. La presencia de hábitos disfuncionales fue independiente al grupo de edad, las diferencias se observaron en relación con el tipo de hábito más frecuente en cada grupo etario.

\section{Facultad de Estudios Superiores Zaragoza, Universidad Nacional Autónoma de México. \\ a. Profesor de Carrera Titular C, División de Estudios de Posgrado e Investigación. Facultad de Estudios Superiores Zaragoza. Universidad Nacional Autónoma de México.}

Correspondencia:

José Francisco Murrieta Pruneda Tenorios 91, casa 24-D Col. Ex Hacienda Coapa, Tlalpan 14300, Ciudad de México, México.

Teléfono: (+55) 56230701.

E-mail: murrieta2010@gmail.com

PALABRAS CLAVES:

Estudio transversal; Preescolares; Infantes; Grupo etario; Hábitos orales disfuncionales.

KEYWORDS:

Cross-Sectional Study, Preschoolers, Infant-children, Age groups, Dysfunctional oral habits. 


\section{ABSTRACT}

Objective: The aim of this study was to compare the frequency of dysfunctional oral habits and their relationship with the age group: infant or preschool.

Material and Methods: A cross-sectional study was carried out in a sample of 269 infant-children and preschoolers with express authorization of their parents. The survey consisted the oral examination and questionnaire application (Cronbach's alpha $=0.832$ ), after intra-calibration of a Dental Surgeon (Kappa $\geq 0.941)$. To measure the reliability of the questionnaire, the value of Cronbach's alpha was calculated and for the associations Pearson's Chi-square.

Results. The habit of biting objects was the most frequent habit (31.2\%), onychophagia and mouth breathing showed statistically significant differences in relation to the group of preschoolers $(\mathrm{p}=0.004 ; \mathrm{p}=0.023)$, while the use of bottle was for the Infant-children group $(\mathrm{p}=0.0001)$.

Conclusions. The frequency of the event had a similar behavior in both infants and preschoolers, the differences observed were related to the type of habit most frequent in each age group.

\section{INTRODUCCIÓN}

Los hábitos son comportamientos que se adquieren por la constante repetición de ciertas acciones presentes en la vida cotidiana ${ }^{1-5}$, en este sentido, los hábitos orales disfuncionales son costumbres o prácticas adquiridas que su repetición constante carece de funcionalidad, los cuales inicialmente pueden realizarse de forma consciente, pero si su actividad se prolonga, puede convertirse en un hábito involuntario que puede provocar alteraciones en el desarrollo orofacial ${ }^{6-11}$. Su frecuencia es variable, ya que han sido observados casos que van desde el $40.7 \%$ en niños colombianos ${ }^{12}$ hasta el $100 \%$ en cubanos $^{13}$, sin que se haya demostrado hasta el momento algún tipo de relación en cuanto al sexo ni a la edad ${ }^{14-18}$, no obstante, con respecto a esta última variable, al parecer el comportamiento epidemiológico de este tipo de hábitos puede distribuirse de forma diferente dependiendo del grupo etario al cual se haga referencia. En cuanto a su comportamiento, se ha observado que tanto el uso prolongado del chupete y del biberón suelen ser más frecuentes en infantes, la onicofagia y la succión digital son los de mayor prevalencia en preescolares y escolares $^{12-17,}$ 19, 21-30. Asimismo, mientras los reportes indican que la onicofagia y la succión digital son los de mayor frecuencia en preescolares, el uso de mamila y del chupete son los de mayor prevalencia en infantes ${ }^{14,19-22}$.

El presente trabajo de investigación tuvo como objetivo comparar la frecuencia de hábitos orales disfuncionales y su relación con dos grupos etarios: preescolar e infantil, inscritos en Centros de Atención y Cuidado Infantil (CACI) del gobierno de la Ciudad de México.

\section{MATERIAL Y MÉTODOS}

\section{Diseño del estudio y participantes}

Se llevó a cabo un estudio descriptivo, en la modalidad transversal, en una muestra por conveniencia inicialmente conformada por 297 niños en edad infantil (incluidos niños de 1 y 2 años) y preescolar (incluidos niños entre 3 y 5 años), inscritos en cinco Centros de Atención y Cuidado Infantil (CACI) de la Ciudad de México. Fueron excluidos del estudio 28 niños $(9.42 \%)$, debido a que sus padres no autorizaron su inclusión en el estudio, porque no estuvieron presentes el día de la recolección de datos, o bien, porque el niño se rehusó al examen bucal, encuestando finalmente a 269 de ellos.

\section{Consideraciones éticas y legales del estudio}

Se contó con la aprobación del proyecto por el Comité de Bioética y Bioseguridad de la Línea de Investigación Dental Oclusión (LIFESZ230506), adscrita a la Facultad de Estudios Superiores Zaragoza, de la Universidad Nacional Autónoma de México. De igual manera, se solicitó el consentimiento informado a los padres o tutores para que él y su hijo pudieran ser incluidos en el estudio. Durante la estandarización del examinador y la encuesta epidemiológica se consideraron las 
disposiciones de la norma técnica de la Secretaría de Salud de México (NOM013SSA2-200635), que contemplan la normatividad para la prevención en la transmisión de infecciones.

\section{Calibración del examinador}

Para la recolección de datos se llevó a cabo la calibración intra-examinador de una Cirujana Dentista, asegurando su confiabilidad por medio del cálculo de concordancias absolutas, relativas y del Coeficiente de kappa de Cohen, con lo cual se garantizó que dichas concordancias no fueran debidas al azar, en todos los casos el valor de kappa fue igual o mayor a 0,941, esto es, un grado casi perfecto de concordancia.

\section{Procedimiento de recolección de datos.}

La encuesta epidemiológica fue llevada a cabo en un aula escolar durante el horario de clases por una Cirujana Dentista, iluminando el campo clínico con una lámpara frontal MOICO®, USA, recargable, con luz blanca de 1000LM, guantes desechables y un espejo dental plano del \#5, sin aumento marca $\mathrm{Hu}$-Friedy®. A cada uno de los padres o tutores se les aplicó un cuestionario, diseñado exprofeso para el estudio, el cual incluye una serie de ítems que permitieron confirmar la presencia de alguno de los hábitos disfuncionales de interés para el estudio.

\section{Variables y valoración clínica}

Los hábitos orales disfuncionales que se estudiaron en los preescolares fueron: $\mathrm{La}$ succión digital, la succión labial, el uso de chupón o mamila, la onicofagia, la protracción lingual, la queilofagia, la mordedura de objetos y la respiración bucal y su distribución por grupo de edad, ya fuera infante o preescolar, y por sexo ${ }^{1-6,12-15,18,21,30}$. Durante el examen clínico se exploraron los dedos verificando su limpieza y evidencia de callosidades; en cuanto a los labios se observó si el superior cubría dos tercios de los incisivos, asimismo, si el cierre de los labios se efectuaba de manera suave sin forzar al inferior. Si el labio inferior se localizaba sin alteraciones en relación con el superior y si mostraba irritación de la piel circundante al labio inferior. El examen de la nariz permitió identificar el contorno de las aletas nasales y observar el sellado de los labios durante el acto de respiración; en cuanto a los dedos, se consideró la situación que guardaban las uñas y las cutículas para verificar la existencia de algún tipo de desgaste o mordedura de estas, o presencia de inflamación o infección; y por último se verificó el contorno, inserción y tonicidad de los músculos buccinadores, orbiculares y borla del mentón.

\section{Métodos estadísticos.}

Para el análisis estadístico se utilizó el paquete estadístico SPSS v.21.0 (IBM, USA) versión para Windows. Para medir la presencia de hábitos orales disfuncionales se calcularon frecuencias absolutas y relativas. Para medir la fiabilidad del cuestionario se calculó el valor del Coeficiente alfa de Cronbach. Para determinar la frecuencia se calcularon frecuencias absolutas y relativas, así como intervalos de confianza. Para medir la asociación entre cada hábito con el grupo etario y el sexo, fue calculado el valor de Chi cuadrada de Pearson. En todos los casos se consideró un nivel de confianza del 95\%. Las tablas fueron diseñadas en Office Microsoft ${ }^{\circledR}$ Excel v.365.

\section{RESULTADOS}

La muestra del estudio estuvo conformada por 269 menores los cuales el $27.9 \%$ pertenecieron al grupo de infantes y $72,1 \%$ al de preescolares, con una representación por sexo del $44.6 \%$ para el femenino y el $55.4 \%$ para el masculino (Tabla I). En relación con el cuestionario, el Coeficiente del alfa de Cronbach resultó ser igual a 0,832, lo que evidenció ser un instrumento bastante homogéneo y fiable de acuerdo con el propósito de este. En relación con la presencia de hábitos disfuncionales, el $58.7 \%$ de los menores presentó al menos uno de estos hábitos, siendo el hábito de morder objetos el que se presentó con mayor frecuencia (31.2\%) 
Tabla I: Distribución de la conformación de la muestra de estudio por grupo etario y sexo

\begin{tabular}{lcccccc} 
& \multicolumn{2}{c}{ Infantes } & \multicolumn{2}{c}{ Preescolares } & \multicolumn{2}{c}{ Total } \\
\hline Sexo & $f$ & $\%$ & $f$ & $\%$ & $f$ & $\%$ \\
Niñas & 40 & 14.9 & 80 & 29.7 & 120 & 44.6 \\
Niños & 35 & 13.0 & 114 & 42.4 & 149 & 55.4 \\
Total & $\mathbf{7 5}$ & $\mathbf{2 7 . 9}$ & $\mathbf{1 9 4}$ & $\mathbf{7 2 . 1}$ & $\mathbf{2 6 9}$ & $\mathbf{1 0 0 . 0}$ \\
\hline & \multicolumn{4}{c}{$\mathrm{f}=$ frecuencia absoluta }
\end{tabular}

Tabla II: Distribución de casos de hábitos disfuncionales.

\begin{tabular}{|c|c|c|c|}
\hline & f & $\%$ & $I_{95 \%}$ \\
\hline \multicolumn{4}{|l|}{$\begin{array}{l}\text { Hábitos } \\
\text { disfuncionales }\end{array}$} \\
\hline Ausente & 111 & 41.3 & {$[39.8-44.2]$} \\
\hline Presente & 158 & 58.7 & {$[53.2-65.6]$} \\
\hline \multicolumn{4}{|l|}{ Biberón } \\
\hline Ausente & 256 & 95.2 & {$[92.7-96.4]$} \\
\hline Presente & 13 & 4.8 & {$[2.3-5.8]$} \\
\hline \multicolumn{4}{|l|}{ Succión digital } \\
\hline Ausente & 242 & 90.0 & {$[88.2-93.5]$} \\
\hline Presente & 27 & 10.0 & {$[6.0-14.0]$} \\
\hline
\end{tabular}

\section{Succión labial}

Ausente

Presente

$248 \quad 92.2 \quad[90.4-94.3]$

$21 \quad 7.8 \quad[5.3-11.0]$

\section{Onicofagia}

Ausente

Presente

$22784.4 \quad[81.9-86.1]$

$42 \quad 15.0 \quad[11.8-20.3]$

\section{Queilofagia}

Ausente

Presente

$250 \quad 92.9 \quad[90.8-93.8]$

$19 \quad 7.1 \quad[5.1-10.2]$

Morder objetos

Ausente

Presente

$185 \quad 68.8 \quad[67.1-69.8]$

$84 \quad 31.2 \quad[26.4-37.3]$

\section{Protracción \\ lingual}

Ausente

Presente

\section{$\begin{array}{lll}263 & 97.8 \quad[95.2-98.6\end{array}$ \\ $\begin{array}{lll}6 & 2.2 \quad[0.04-4.2]\end{array}$}

\section{Respiración Bucal}

Ausente

Presente

$222 \quad 82.5 \quad[80.7-84.6]$

$47 \quad 17.5 \quad[13.6-22.1]$

Total

$269 \quad 100$

$\mathrm{f}=$ frecuencia absoluta. $\mathrm{IC}_{95 \%}=$ Intervalo de confianza al 95\% en comparación con los demás, seguido de la onicofagia $(15 \%)$ y respiración bucal nocturna (11.9\%) (Tabla II). En cuanto a la distribución de hábitos disfuncionales por grupo etario, se observó que fueron más frecuentes en el grupo de preescolares $(42.8 \%)$ en comparación con los infantes (16\%) (Tabla III). El comportamiento de cada uno de estos hábitos mostró que el relacionado con morder objetos, la succión labial, la protracción lingual, la succión digital y la respiración bucal fueron más frecuentes en preescolares en comparación con lo observado en niños del grupo de infantes, no obstante, ninguna de esas diferencias fue estadísticamente significativas $\quad(p=0.294 ; p=0.061 ; p=0.536$; $\mathrm{p}=0.116$ y $\mathrm{p}=0.142$ ). El hábito del uso de biberón se presentó con mayor frecuencia en infantes, condición que resultó ser estadísticamente significativa $(p=0.0001)$. En cuanto a la onicofagia y la queilofagia fueron más frecuentes en preescolares, en estos dos casos las diferencias observadas sí resultaron estar asociadas al grupo etario $(p=0.004$ y $\mathrm{p}=0.023$ ) (Tabla IV).

En cuanto a la frecuencia de hábitos disfuncionales por sexo, se observó que predominó más en el sexo masculino (34.6\%), así como en cada uno de los diferentes tipos de hábitos disfuncionales estudiados, no encontrándose diferencias estadísticamente significativas en ninguno de los casos: hábito de morder objetos $(\mathrm{p}=0.237)$; onicofagia $(\mathrm{p}=0.069)$; queilofagia $(\mathrm{p}=0.866)$; protracción lingual $(\mathrm{p}=0.788)$; succión digital $(\mathrm{p}=0.404)$; respiración bucal ( $\mathrm{p}=0.064)$; así como en los casos de uso biberón $(\mathrm{p}=0.648)($ Tabla V).

\section{DISCUSIÓN}

De acuerdo con los resultados obtenidos, la prevalencia de hábitos bucales disfuncionales, fue menor a lo reportado en poblaciones similares por Mendoza et al. ${ }^{14}$, Chumi et al. ${ }^{7} \mathrm{y}$ Aróstica et $a l .{ }^{4}$ pero mayor a lo reportado por Lopes et al. ${ }^{15}$ En cuanto su frecuencia por grupo etario y sexo, se observó que predominó más en preescolares y en hombres, diferente a lo reportado por Al-Sadhan et al. ${ }^{2}$ y por Pacheco et $a l .{ }^{3}$ lo que pone en evidencia que las condiciones del entorno y de comportamiento de 
Tabla III: Distribución de casos de hábitos disfuncionales por grupo etario

\begin{tabular}{|c|c|c|c|c|c|c|}
\hline & \multicolumn{2}{|c|}{ Ausente } & \multicolumn{2}{|c|}{ Presente } & \multicolumn{2}{|c|}{ Total } \\
\hline & $f$ & $\%$ & $f$ & $\%$ & $f$ & $\%$ \\
\hline Infantes & 32 & 11.9 & 43 & 16.0 & 75 & 27.9 \\
\hline $\mathrm{IC}_{95 \%}$ & \multicolumn{2}{|c|}{$[9.7-12.3]$} & \multicolumn{2}{|c|}{ [11.3 -18.4] } & & \\
\hline Preescolares & 79 & 29.4 & 115 & 42.8 & 194 & 72.1 \\
\hline $\mathrm{IC}_{95 \%}$ & \multicolumn{2}{|c|}{$[27.1-31.6]$} & \multicolumn{2}{|c|}{ [36.3 -49.0] } & & \\
\hline Total & 111 & 41.3 & 158 & 58.7 & 269 & 100 \\
\hline
\end{tabular}

Tabla IV: Distribución de hábitos disfuncionales, por grupo etario

\begin{tabular}{|c|c|c|c|c|c|c|}
\hline & \multicolumn{2}{|c|}{ Ausente } & \multicolumn{2}{|c|}{ Presente } & \multirow[t]{2}{*}{$\mathrm{IC}_{95 \%}$} & \multirow[t]{2}{*}{ Significancia } \\
\hline & f & $\%$ & $f$ & $\%$ & & \\
\hline \multicolumn{7}{|l|}{ Biberón } \\
\hline Infantes & 65 & 24.2 & 10 & 3.7 & {$[21.3-26.4] ;[1.09-4.60]$} & \\
\hline Preescolares & 191 & 71.0 & 3 & 1.1 & {$[11.8-16.7] ;[0.05-1.80]$} & $p=0.0001^{*}$ \\
\hline \multicolumn{7}{|c|}{ Succión labial } \\
\hline Infantes & 73 & 27.1 & 2 & 0.7 & {$[24.8-28.7] ;[0.09-0.98]$} & \\
\hline Preescolares & 175 & 65.1 & 19 & 7.1 & {$[62.4-67.1] ;[3.80-9.10]$} & $p=0.061$ \\
\hline \multicolumn{7}{|l|}{ Onicofagia } \\
\hline Infantes & 71 & 26.4 & 4 & 1.5 & {$[24.6-27.7] ;[0.66-2.10]$} & \\
\hline Preescolares & 156 & 58.0 & 38 & 14.1 & {$[56.2-60.1] ;[11.8-16.7]$} & $p=0.004^{*}$ \\
\hline \multicolumn{7}{|l|}{ Queilofagia } \\
\hline Infantes & 74 & 27.5 & 1 & 0.4 & {$[25.3-29.2] ;[0.03-0.95]$} & \\
\hline Preescolares & 176 & 65.4 & 18 & 6.7 & {$[63.9-67.3] ;[5.20-8.10]$} & $p=0.023^{*}$ \\
\hline \multicolumn{7}{|c|}{ Morder objetos } \\
\hline Infantes & 48 & 17.8 & 27 & 10.0 & [15.9-16.7]; [8.30-12.40] & \\
\hline Preescolares & 137 & 50.9 & 57 & 21.2 & {$[48.6-53.0] ;[17.2-24.8]$} & $p=0.294$ \\
\hline \multicolumn{7}{|c|}{ Protracción lingual } \\
\hline Infantes & 74 & 27.5 & 1 & 0.4 & {$[24.8-29.7] ;[0.02-0.93]$} & \\
\hline Preescolares & 189 & 70.3 & 5 & 1.9 & {$[68.6-72.5] ;[0.08-2.70]$} & $p=0.536$ \\
\hline \multicolumn{7}{|c|}{ Succión digital } \\
\hline Infantes & 64 & 23.8 & 11 & 4.1 & [21.9-24.7]; [1.3 - 5.9] & \\
\hline Preescolares & 178 & 66.2 & 16 & 5.9 & {$[64.4-67.9] ;[2.4-7.3]$} & $p=0.116$ \\
\hline \multicolumn{7}{|c|}{ Respiración bucal } \\
\hline Infantes & 66 & 24.5 & 9 & 3.3 & {$[22.1-25.8] ;[2.10-4.60]$} & \\
\hline Preescolares & 156 & 58.0 & 38 & 14.1 & {$[56.2-60.1] ;[11.0-16.3]$} & $p=0.142$ \\
\hline
\end{tabular}


Tabla V: Distribución de hábitos disfuncionales, por grupo sexo.

\begin{tabular}{cccccc} 
Ausente & Presente & & $\mathrm{IC}$ 95\% & Significancia \\
\hline$f$ & $\%$ & $f$ & $\%$ & &
\end{tabular}

\section{Hábitos disfuncionales}

Femenino

Masculino

\section{Biberón}

Femenino

Masculino

\section{Succión labial}

Femenino

Masculino

\section{Onicofagia}

Femenino

Masculino

\section{Queilofagia}

Femenino

Masculino

\section{Morder objetos}

Femenino

Masculino

\section{Protracción lingual}

\section{Femenino}

Masculino

\section{Succión digital}

Femenino

Masculino

\section{Respiración bucal}

Femenino

Masculino $\begin{array}{lllll}55 & 20.4 & 65 & 24.2 & {[19.2-21.8] ;[21.3-26.9]}\end{array}$

$\begin{array}{llllll}56 & 20.8 & 93 & 34.6 & {[19.4-21.6] ;[30.6-36.9]} & p=0.215\end{array}$ $\begin{array}{lllll}115 & 42.8 & 5 & 1.9 & {[41.5-44.0] ;[0.08-2.6]}\end{array}$

$\begin{array}{lllll}141 & 52.4 & 8 & 3.0 & {[50.3-54.8] ;[1.15-4.2] \quad p=0.648}\end{array}$

$$
\begin{array}{rrrrr}
111 & 41.3 & 9 & 3.3 & {[39.7-43.6] ;[0.09-5.2]}
\end{array}
$$$$
\begin{array}{lllll}
137 & 50.9 & 12 & 4.5 & {[48.4-52.7] ;[2.7-6.10]}
\end{array}
$$$$
p=0.866
$$

$\begin{array}{lll}100 & 37.2 & 20\end{array}$

$7.4 \quad[36.1-38.3] ;[5.9-8.6]$

$127 \quad 47.2 \quad 22$

$8.2 \quad[45.3-48.8] ;[6.4-9.7]$

$p=0.669$

$\begin{array}{rrrrr}114 & 42.4 & 6 & 2.2 & {[40.6-43.9] ;[1.4-3.1]}\end{array}$

$p=0.866$

$87 \quad 32.3 \quad 33 \quad 12.3 \quad[31.2-33.5] ;[8.3-14.4]$

$\begin{array}{llllll}98 & 36.4 & 51 & 19.0 & {[34.4-37.8] ;[17.2-24.8]} & p=0.237\end{array}$

$117 \quad 43.5 \quad 3 \quad 1.1 \quad[41.5-44.0] ;[0.03-0.98]$

$\begin{array}{llllll}146 & 54.0 & 3 & 1.1 & {[52.8-56.2] ;[0.06-2.3] \quad p=0.788}\end{array}$

$110 \quad 40.9 \quad 10 \quad 3.7 \quad[38.9-42.3] ;[2.3-4.9]$

$\begin{array}{llllll}132 & 49.1 & 17 & 6.3 & {[48.2-51.0] ;[4.4-7.8] \quad p=0.404}\end{array}$

$105 \quad 39.1 \quad 15 \quad 5.6 \quad[37.7-41.1] ;[4.1-7.2]$

$\begin{array}{llllll}117 & 43.4 & 32 & 11.9 & {[41.3-43.9] ;[10.3-12.8] \quad p=0.064}\end{array}$ 
cada sujeto ${ }^{1,3-5}$. Su relevancia desde el punto de vista clínico está relacionada con el hecho de que estos pueden ejercer acciones mecánicas y funcionales que provocan alteraciones en el sistema estomatognático, razón por la cual son considerados como factor de riesgo que pueden alterar el establecimiento y desarrollo de la oclusión dental. En cuanto a su frecuencia por grupo etario, se observó más en preescolares con respecto a infantes, el riesgo a desarrollarlos tal vez no cambie, pero sí la probabilidad de que ciertos hábitos tengan mayor posibilidad de que sean adoptados, debido probablemente a las condiciones de su entorno familiar, social y cultural bajo los cuales crecen. Así, no fue una casualidad que el uso del biberón fuera más frecuente en infantes en comparación con preescolares, ya que es más probable que un infante en comparación de un preescolar presente este hábito. En relación con el sexo, a pesar de que los hombres presentaron este tipo de hábitos con mayor frecuencia, tal comportamiento no fue suficiente para intuir que esta variable tuviera alguna relevancia en el desarrollo de cualquier hábito disfuncional, de tal forma, que no importó que fuera niña o niño, en ambos casos al parecer esta condición no favoreció que se presentara con mayor frecuencia en alguna de las dos categorías ${ }^{14-18}$. El hábito de morder objetos fue el más frecuente, comportamiento que resultó mayor a lo observado por Chumi et al. ${ }^{7}$ pero menor a lo reportado por Arias et $a l .{ }^{1}$ La relevancia de este dato radica en el riesgo que se presenta dicho hábito en la población para afectar el desarrollo de la oclusión dental en la dentición secundaria, alterando las condiciones de la relación que guardan los dientes entre sí en el sextante anterior, el cual puede manifestarse en un resalte anterior aumentado ${ }^{13,16-17}$. El hábito del uso de biberón resultó mayor a lo reportado por Mendoza et al..$^{14}$ pero menor a lo reportado por Orengul et al. ${ }^{23}$, se observó con mayor frecuencia en infantes, mostrando evidencia estadísticamente significativa. Su relevancia radica en el hecho de que el biberón al ser más largo y grueso desplaza la lengua hacia el piso de la boca lo que impide un movimiento anterior mandibular adecuado y provoca que permanezca en una posición distal, provocando retraso del crecimiento óseo y malposiciones dentales como: mordida cruzada, mordida abierta y overjet y overbite incrementados $^{23}$. La succión labial fue menor a lo reportado por Mendoza et al. ${ }^{14}$ pero mayor a lo reportado por Arias et al. ${ }^{1}$. De acuerdo con el grupo etario, predominó en el preescolar y en el sexo masculino, sin embargo, estas diferencias no resultaron ser estadísticamente significativas razón por la cual esta condición no resultó relevante. El hábito de onicofagia resultó menor a lo reportado por Orozco et al. ${ }^{77}$ pero mayor a lo reportado por Chumi et al. ${ }^{7}$. Se presentó con mayor frecuencia en la población preescolar, evidencia estadísticamente significativa. Este comportamiento puede deberse a que la onicofagia ha sido reportada como un hábito que se presenta con mayor frecuencia en niños y adultos jóvenes, se presenta por situaciones muy particulares, como una forma de atraer la atención, si se encuentran expuestos a un entorno familiar violento, a la falta de atención de los padres, a la falta de madurez emocional, a los cambios constantes en el ambiente familiar, o bien, a modo de imitación, por lo tanto cada individuo tiene probabilidades diferentes para desarrollarlo. Su relevancia clínica consiste en que puede conducir a una reducción de la fuerza de los músculos masticatorios, desgaste de los bordes incisales, rotación y movilidad dental y maloclusión principalmente en dientes anteriores $^{1-3,7-8}$. El hábito de queilofagia se presentó con menor frecuencia, en comparación a lo reportado por Kamdar et al. ${ }^{18}$ pero mayor a lo reportado por Rodríguez et al. ${ }^{22}$, ligeramente mayor en el sexo masculino pero con predominio en el grupo de preescolares, diferencia que resultó ser estadísticamente significativa, no obstante, debe tenerse en cuenta que este hábito no necesariamente es un factor causal de una maloclusión dental sino una consecuencia de ella, y por lo tanto, su presencia no provoca tanta preocupación como el tipo de maloclusión que presenta el niño. El hábito de protracción lingual tuvo un comportamiento menor a lo reportado por Mendoza et al. ${ }^{14}$ ligeramente más frecuente en preescolares con respecto a infantes. Este hábito es importante 
para la oclusión ya que la puede alterar debido a que las fuerzas que se generan durante la ejecución de este hábito son suficientes para provocar modificaciones dentoalveolares que orientan hacia una mordida abierta y cruzada anterior o posterior. En cuanto al hábito de succión digital, mostró una frecuencia menor a lo reportado por Mendoza et al. ${ }^{14}$ y por Doğramac1 et al. ${ }^{9}$. Se observó que los preescolares realizaban el hábito con mayor frecuencia que los infantes, predominando en el sexo masculino, sin embargo, estas diferencias no resultaron ser significativas es decir no importó el rango de edad ni que fuera niño o niña, en ambos casos al parecer todos presentaron un riesgo similar para desarrollar este hábito. En relación con el hábito de respiración bucal resultó menor a lo reportado por Arias A. et al. ${ }^{1}$, de acuerdo con la edad, se observó que la prevalencia fue ligeramente mayor en el grupo de preescolares y predominó en el sexo masculino, sin que se encontrara diferencias significativas entre estas variables. La importancia de que se encontró presente este hábito en la muestra de estudio radica en que este hábito es considerado normal hasta los tres años ${ }^{27-28}$, no obstante, si se prolonga las alteraciones en las estructuras podrían ser irreversibles, las cuales pueden manifestarse en la vestibuloversión de incisivos superiores, linguoversión de incisivos inferiores, diastemas, retrognatismo mandibular, bóveda palatina profunda, mordida abierta anterior o posterior (la cual dependerá de los dedos succionados y su dirección), micrognatismo transversal, mordida cruzada e interferencia en la erupción y posición dental ${ }^{26-29}$.

\section{CONCLUSIONES}

La presencia de hábitos disfuncionales fue independiente al grupo de edad, las diferencias se observaron en relación con el tipo de hábito más frecuente en cada grupo etario. Se resalta la importancia de llevar a cabo acciones educativas con los padres sobre dichos hábitos, con el propósito de eliminarlos para evitar secuelas, o bien, para prevenir su aparición en aquellos niños que no los presentaron.

\section{CONFLICTOS DE INTERÉS}

Sin clonflictos de interés

\section{REFERENCIAS}

[1] Arias AA, Espinal BG, Ponce PM, Posada LA, Nava CJ, Salcedo OB. Frecuencia de hábitos orales relacionados con la maloclusión en pacientes de 4 a 12 años: estudio comparativo entre San Luis de Potosí-México y Medellín -Colombia, 2016. Rev Nac Odontol. 2017;14(26):2357-4607. DOI: 10.16925/od.v13i26.1814

[2] Al-Sadhan SA, Al-Jobair AM. Oral habits, dental trauma, and occlusal characteristics among 4- to 12-year-old institutionalized orphan children in Riyadh, Saudi Arabia. Spec Care Dent. 2017;37(1):10-8.

[3] Pacheco P, Hernández A. Relación entre los hábitos bucales deformantes y desórdenes en el plano emocional y psicológico. Oral 2019. 2019;20(62):1698704.

[4] Aróstica N, Carrillo G, Cueto A, Mariño, D, Teresa Jofré $T$. Prevalence of malocclusions and dysfunctional oral habits in preschool children of municipal establishments in Viña del Mar. J Oral Res 2020; 9(4):271-9.

DOI:10.17126/joralres.2020.068

[5] Alves FT, Wambier DS, Alvarez JA, da Rocha JF, Kummer TR, de Castro VC, et al. Children using day nurseries' facilities can be associated with more risk to nonnutritive sucking habits. J Contemp Dent Pract. 2016;17(9):721-7.

[6] Rekka NI, Sathiyawathie RS, Felcita S. Correlation between oral habits causing malocclusion in children. Drug Invent Today. 2019;11(4):822-4. DOI:10.5005/jpjournals-10024-1919

[7] Chumi R. Pinos P. Prevalencia de hábitos orales, en niños de 3 a 9 años, como consecuencia de la migración de los padres al extranjero, en la comunidad Callazay, Parroquia Mariano Moreno del Cantón Gualaceo Provincia del Azuay - Ecuador, año 2014. Revista latin ortod odont. 2015. Disponible

en: https://www.ortodoncia.ws/publicaciones/20 15/art-18/ 
[8] Ling $\mathrm{HB}$, Sum FH, Zhang L, Yeung CW, $\mathrm{Li} \mathrm{KY}$, Wong HM. The association between nutritive, non-nutritive sucking habits and primary dental occlusion. BMC Oral Health. 2018;18(1):1-10. DOI:10.1186/s12903-018-0610-7

[9] Doğramacı EJ, Rossi G. Establishing the association between nonnutritive sucking behavior and malocclusions: A systematic review and meta-analysis. J Amer Dental Asso. 2016; 147. 926-34. DOI:10.1016/j.adaj.2016.08.018

[10] Machado S, Manzanares M, FerreiraMoreira J, Ferreira J, Rompante $\mathrm{P}$, Ustrell J. A sample of non-nutritive sucking habits (pacifier and digit) in portuguese children and its relationship with the molar classes of angle. J Clin Exp Dent. 2018;10(12):e1161-6. DOI:10.4317/jced.55284

[11] Chen X, Xia B, Ge L. Effects of breastfeeding duration, bottle-feeding duration and non-nutritive sucking habits on the occlusal characteristics of primary dentition. BMC Pediatr. 2015;15(1). DOI:10.1186/s12887-015-0364-1

[12] Meneses EJ. Epidemiological Profile of Static Occlusion and Oral Habits in a Group of schoolchildren in Medellin, Colombia. Rev Nac Odont. 2018; 12(22): 67. DOI:10.16925/od.v12i22.1207

[13] Gómez YA. Factores de riesgo asociados con anomalías de oclusión en dentición temporal. Rev Cuba Scielo. 2015;19(1):66-76.

[14] Mendoza L. Meléndez AF. Ortiz R. Fernández A. Prevalencia de las maloclusiones asociada con hábitos bucales nocivos en una muestra de mexicanos. Rev Mex Ortod. 2014; 2(4): 220-7.

[15] Lopes FM, Espasa SE, Rodrigues SC, Butini OL, Ustrell T. Non-nutritive sucking habits and their effects on the occlusion in the deciduous dentition in children PubMed. Eur J Paediatr Dent. 2016: https://pubmed.ncbi.nlm.nih.gov/2804531 8/

[16] Sharma S. Bansal A. Asopa K. Prevalence of Oral Habits among Eleven to Thirteen Years Old Children in Jaipur.
Int J Clin Pediatr Dent 2015; 8(3):208-10. https://www.ijcpd.com/doi/pdf/10.5005/jpjournals-10005-1314

[17] Kolawole KA, Folayan MO, Agbaje $\mathrm{HO}$, Oyedele TA, Onyejaka NK, Oziegbe EO. Oral habits and malocclusion in children resident in Ile-Ife Nigeria. Eur Arch Paediatr Dent. 2019;20(3):257-65. DOI:10.1007/s40368-018-0391-3

[18] Kamdar RJ, Al-Shahrani I. Damaging oral habits. J Int Oral Heal JIOH. 2015;7(4):857.

[19] Antony TL, Priya VV, Gayathri R. Awareness on thumb sucking and pacifier and its effect on child's teeth. Drug Invent Today. 2019;12(7):1368-71.

[20] Onyejaka NK, Kolawole KA, Folayan MO. Habit-breaking methods employed by mothers of children with nonnutritive sucking habits resident in suburban Nigeria. Indian J Dent Res. 2017;29(2):212-6. DOI:10.4103/ijdr.ijdr_404_16

[21] Fonseca GM. Vaudagnar R. Galván F. Queilofagia como evidencia para la perfilación e investigación criminal. Rev Arg Morfol. 2013; 11(1):12-6.

[22] Rodríguez N, Horta $M$, Vences $N$. Tratamiento de hábitos deformantes bucales en niños de 4 a 13 años con auriculoterapia. Archivo Médico de Camagüey [Internet]. 2017;21(6):740-52.

[23] Orengul AC, Tarakcioglu MC, Gormez V, Akkoyun S, Aliyeva N. Duration of Breastfeeding, Bottle-Feeding, and Dysfunctional Oral Habits in Relation to Anxiety Disorders among Children. Breastfeed Med. 2019;14(1):57-62. DOI:10.1089/bfm.2018.0013

[24] Gür K, Erol S, İncir N. The effectiveness of a nail-biting prevention program among primary school students. J Spec Pediatr Nurs. 2018;23(3):1-9.

DOI:10.1111/jspn.12219

[25] Winebrake JP, Grover K, Halteh P, Lipner SR. Pediatric Onychophagia: A SurveyBased Study of Prevalence, Etiologies, and Co-Morbidities. Am J Clin Dermatol. 2018;19(6):887-91. DOI:10.1007/s40257018-0386-1

[26] Milanesi JM, Berwig LC, Marquezan M, Schuch LH, de Moraes AB, da Silva AM. 
Variables associated with mouth breathing diagnosis in children based on a multidisciplinary assessment. Codas. 2018;30(4):1-9. DOI: 10.1590/23171782/20182017071

[27] Grippaudo C, Paolantonio EG, Antonini G, Saulle R, La Torre G, Deli R. Association between oral habits, mouth breathing and malocclusion. Acta Otorhinolaryngol Ital. 2016;36(5):386-94. DOI:10.14639\%2F0392-100X-770

[28] Fraga WS, Seixas VM, Santos JC, Paranhos LR, César CP. Mouth breathing in children and its impact in dental malocclusion: A systematic review of observational studies. Minerva Stomatol. 2018;67(3):129-38. DOI: 10.23736 / S0026-4970.18.04015-3

[29] Silva GA, Bulnes RM, Rodríguez LV. Prevalencia de hábito de respiración oral como factor etiológico de maloclusión en escolares del Centro, Tabasco. Rev ADM. 2014;71(6):285-9.

[30] Arocha AA, Aranda GMS, Pérez PY, et al. Maloclusiones y hábitos bucales deformantes en escolares con dentición mixta temprana. MediSan. 2016;20(04):426-32.

\section{CÓMO CITAR ESTE ARTÍCULO}

Murrieta J, Ramírez M, Salgado L, Salamanca R. Frecuencia de hábitos bucales parafuncionales y su relación con el grupo etario. Appli Sci Dent. 2021:1(1);1-10

DOI: 10.22370/asd.2021.1.1.2529
Applied Sciences in Dentistry, revista científica de la Facultad de Odontología de la Universidad de Valparaíso, de Acceso Abierto y de Publicación Continua.

Son aceptados trabajos originales e inéditos, abarcando cartas al editor, comunicaciones breves, artículos de investigación, casos clínicos y revisiones bibliográficas.

Correo electrónico de contacto:

contacto.asdj@uv.cl

editor.asdj@uv.cl

Página Web:

https://revistas.uv.cl/index.php/asid

Redes Sociales

Instagram @asd.journal

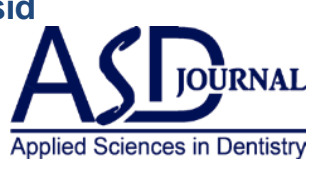

